\title{
CNN1 regulates the DKK1/Wnt/ $\beta$-catenin/c-myc signaling pathway by activating TIMP2 to inhibit the invasion, migration and EMT of lung squamous cell carcinoma cells
}

\author{
WUSHENG LIU ${ }^{1}$, XIAOGANG FU ${ }^{2}$ and RUMEI LI ${ }^{3}$ \\ ${ }^{1}$ Department of Respiratory Medicine, The Affiliated Xinyu Hospital of Nanchang University; \\ Departments of ${ }^{2}$ Respiratory Medicine and ${ }^{3}$ Endocrinology, Xinyu People's Hospital, Xinyu, Jiangxi 338000, P.R. China
}

Received October 23, 2020; Accepted February 12, 2021

DOI: $10.3892 / \mathrm{etm} .2021 .10287$

\begin{abstract}
The present study aimed to investigate the effect of calponin $1(\mathrm{CNN} 1)$ on the invasion and migration of lung squamous cell carcinoma (LUSC) cells and the associations between CNN1, tissue inhibitor of metalloproteinases 2 (TIMP2), Dickkopf-1 (DKK1) and the Wnt/ $\beta$-catenin/c-myc signaling pathway. The expression levels of CNN1 and TIMP2 in LUSC cells and the association between CNN1 and TIMP2 were predicted using the GEPIA database. The cells were transiently transfected to overexpress CNN1, which resulted in inhibition of DKK1 and TIMP2 expression levels. Wound healing and Transwell assays were used to detect the invasive and migratory abilities of LUSC cells. Reverse transcription-quantitative PCR and western blotting were used to investigate the expression levels of CNN1, MMP2, MMP9, E-cadherin, N-cadherin (N-cad), SLUG, DKK1, $\beta$-catenin and c-myc. The expression levels of $\mathrm{N}$-cad were detected using immunofluorescence staining. The results indicated that overexpression of CNN1 inhibited the invasion and migration of NCI-H2170 cells. Inhibition of DKK1 reversed this change and the expression levels of $\beta$-catenin and c-myc were upregulated, whereas the expression levels of DKK1 were downregulated with a concomitant inhibition of TIMP2. In summary, these results demonstrated that CNN1 regulated the DKK1/Wnt/ $\beta$-catenin/c-myc signaling pathway by activating TIMP2 to inhibit the invasion, migration and epithelial-to-mesenchymal transition of LUSC cells.
\end{abstract}

\section{Introduction}

Lung cancer ranks first in incidence and mortality among malignant tumors worldwide. The incidence of malignant lung

Correspondence to: Dr Wusheng Liu, Department of Respiratory Medicine, The Affiliated Xinyu Hospital of Nanchang University, 369 North Xinxin Road, Yushui, Xinyu, Jiangxi 338000, P.R. China E-mail: liuwusheng99@126.com

Key words: lung squamous cell carcinoma, non-small cell lung cancer, calponin 1 , invasion, migration tumors has increased over the last decades and this type of cancer is responsible for $>1.3$ million deaths worldwide annually (1). Lung tumors are one of the most frequent malignant tumors types in China $(2,3)$. Lung cancer is mainly divided into non-small cell lung cancer (NSCLC) and small cell lung cancer, with NSCLC accounting for $\sim 80 \%$ of all cases (4). The two main subtypes of NSCLC are lung adenocarcinoma and lung squamous cell carcinoma (LUSC), and LUSC is insidious and develops rapidly (5). A subset of patients with LUSC do not have the opportunity to receive radical surgery and, consequently, the survival rate of patients is low (6). It has been shown that $<5 \%$ of patients survive 5 years following chemotherapy treatment (7). Therefore, the identification of new and effective treatments for this disease is crucial.

Basic calponin (CNN) or CNN1 (also referred to as calmodulin 1) is a marker for the differentiation of cardiac and smooth muscle (8). It is one of three subtypes of CNN and is encoded by a gene on human chromosome 19 (19p13.2-p13.1) (8). The expression levels of CNN1 are abnormal under various pathological conditions, such as abnormal gastrointestinal motility (9) and hypoxia (10). In addition, a number of studies have shown that CNN1 is expressed at low levels in a variety of tumor tissue types, such as malignant melanoma (11), hepatocellular carcinoma (12), ovarian cancer (13) and breast cancer (14). However, to the best of our knowledge, the association between CNN1 and LUSC has not been reported previously. In the present study, the effects of CNN1 on the invasive and migratory abilities of LUSC cells were investigated.

The Wnt/ $\beta$-catenin signaling pathway is involved in various crucial cellular functions, such as stem cell regeneration and organogenesis (15). Wnt activation has been observed in various types of malignant tumor, including in the breast, lung and hematopoietic system, and has been shown to contribute to tumor recurrence (16). In NSCLC, it has been reported that targeting the negative regulators of Wnt signaling for degradation to increase $\beta$-catenin-mediated Wnt activity may lead to the maintenance of lung cancer 'stemness' (17). The activation of the Wnt/ $\beta$-catenin signaling pathway promotes tumor growth, metastasis and epithelial-to-mesenchymal transition (EMT) of NSCLC cells (18). Dickkopf-1 (DKK1) is a member of the DKK protein family, which is a secretory protein that 
acts as an inhibitor of the extracellular Wnt signal transduction pathway (19). c-myc is one of the target genes of $\mathrm{Wnt} / \beta$-catenin pathway and can be activated by the transcription factor, $\beta$-catenin, which enters the nucleus to regulate target genes expression upon being stabilized by Wnt binding (20). Notably, c-myc functions as a critical oncogene and has been shown to be implicated in enhancing the aggressiveness of various cancer types, including lung cancer (21). The present study aimed to investigate whether the DKK1/Wnt/ $\beta$-catenin/c-myc signaling pathway participated in the effect of CNN1 on LUSC.

\section{Materials and methods}

Cell culture and transfection. The LUSC cell lines NCI-H520, SK-MES-1 and NCI-H2170 were obtained from the Cell Bank of Type Culture Collection of the Chinese Academy of Sciences. All cells were cultured in DMEM (Gibco; Thermo Fisher Scientific, Inc.) supplemented with 10\% FBS (Gibco; Thermo Fisher Scientific, Inc.) and maintained at $37^{\circ} \mathrm{C}$ and $5 \% \mathrm{CO}_{2}$. Overexpression (OE) vectors for CNN1 and knockdown vectors for DKK1 and tissue inhibitor of metalloproteinases 2 (TIMP2) [OE-negative control (NC; empty pcDNA3.1), OE-CNN1 (pcDNA3.1-CNN1), short hairpin RNA (shRNA)-NC, shRNA-TIMP2 and shRNA-DKK1] were purchased from Biomics Biotechnologies. Cell transfection in NCI-H2170 cells was performed using Lipofectamine ${ }^{\circledR} 2000$ (Invitrogen; Thermo Fisher Scientific, Inc.) according to the manufacturer's instructions. Briefly, Lipofectamine 2000 was mixed with $20 \mu \mathrm{g}$ plasmids, which was then added to the cells at $70-80 \%$ confluence and incubated for $6 \mathrm{~h}$ at $37^{\circ} \mathrm{C}$. The transfection efficiency in cells was measured using reverse transcription-quantitative PCR (RT-qPCR). At $48 \mathrm{~h}$ post-transfection, the transfection efficacy was validated using RT-qPCR and successfully transfected cells were selected for subsequent experiments.

Dynamic analysis of GEPIA gene expression profile data. GEPIA (http://gepia.cancer-pku.Cn/index.html) is a website for cancer data mining. Using RNA sequencing data for CNN1 and TIMP2 from tumor and normal samples, the database was used to analyze the expression levels and association between CNN1 and TIMP2 in tumor samples.

$R T-q P C R$. Total RNA was extracted using TRIzol ${ }^{\circledR}$ (Invitrogen: Thermo Fisher Scientific, Inc.). The mRNA sequence was reversed-transcribed into cDNA using a reverse transcriptase (HiScript II Reverse Transcriptase; Vazyme Biotech Co., Ltd.) and the reaction conditions were set as follows: $42^{\circ} \mathrm{C}$ for $\sim 1 \mathrm{~h}$ and $90^{\circ} \mathrm{C}$ for $5 \mathrm{~min}$. The expression levels of CNN1, MMP2, MMP9, DKK1 and TIMP2 were subsequently detected using qPCR by SYBR-Green ROX-mix (ChamQ SYBR qPCR Master Mix; Vazyme Biotech Co., Ltd.). The PCR reaction procedure was as follows: $5 \mathrm{~min}$ at $95^{\circ} \mathrm{C}$, with 40 cycles of $30 \mathrm{sec}$ at $95^{\circ} \mathrm{C}$ and $45 \mathrm{sec}$ at $65^{\circ} \mathrm{C}$. Expression levels of target genes were analyzed using the $2^{-\Delta \Delta \mathrm{Cq}}$ method (22) after being normalized to the expression levels of GAPDH. The primer sequences used were as follows: MMP2 forward, 5'-GATACCCCTTTGACGGTAAGGA-3' and reverse, 5'-CCTTCTCCCAAGGTCCATAGC-3'; MMP9 forward, 5'-GGGACGCAGACATCGTCATC-3' and reverse,
5'-TCGTCATCGTCGAAATGGGC-3'; CNN1 forward, 5'-TGAAGAAGATCAATGAGTCAACC-3' and reverse, 5'-CGTTCACCTTGTTTCCTTTCG-3'; DKK1 forward, 5'-GAAGAGTGTTAAAGGTTTTTTTTTATGTAT-3' and reverse, 5'-CCAAAАTCCTAАCTACAAAAAACACA-3'; TIMP2 forward, 5'-CTCTGATTTGGTCGTATTGGG-3' and reverse, 5'-TGGAAGATGGTGATGGGATT-3'; and GAPDH forward, 5'-GCAACCGGGAAGGAAATGAATG-3' and reverse, 5'-CCCAATACGACCAAATCAGAGA-3'.

Western blotting. The cells were harvested and total protein was extracted using RIPA lysis buffer (Beyotime Institute of Biotechnology). Protease inhibitors were added (Beyotime Institute of Biotechnology) to the lysis buffer (1:100). The lysates were centrifuged at $4^{\circ} \mathrm{C}, 850 \mathrm{x}$ g for $15 \mathrm{~min}$. The supernatant was collected and mixed with a loading buffer (Beyotime Institute of Biotechnology) containing $100 \mathrm{mM}$ dithiothreitol. Total protein was quantified using a Protein Concentration Determination BCA kit (Beyotime Institute of Biotechnology) and the proteins (30 $\mu \mathrm{g} / \mathrm{lane})$ were separated via SDS-PAGE (15\%). The separated proteins were subsequently transferred onto PVDF membranes (EMD Millipore) and blocked using 5\% BSA (Beyotime Institute of Biotechnology) at room temperature for $2 \mathrm{~h}$. The membranes were incubated with the following primary antibodies at $4^{\circ} \mathrm{C}$ overnight: Anti-CNN1 (cat. no. ab46794; 1:1,000 dilution), anti-MMP2 (cat. no. ab92536; 1:1,000 dilution), anti-MMP9 (cat. no. ab76003; 1:1,000 dilution), anti-E-cadherin (E-cad; cat. no. ab40772; 1:1,000 dilution), anti-N-cadherin (N-cad; cat. no. ab245117; 1:1,000 dilution), anti-SLUG (cat. no. ab51772; 1:1,000 dilution), anti-DKK1 (cat. no. ab109416; $1: 1,000$ dilution), anti- $\beta$-catenin (cat. no. ab32572; 1:1,000 dilution), anti-c-myc (cat. no. ab32072; 1:1,000 dilution) and anti-GAPDH (cat. no. ab8245; 1:10,000 dilution). All antibodies were purchased from Abcam. Following the primary incubation, the membranes were washed with TBS containing Tween-20 (0.1\%) and incubated at room temperature for $1.5 \mathrm{~h}$ with a horseradish peroxidase-conjugated donkey anti-rabbit IgG secondary antibody (cat. no. SA00001-9; 1:5,000 dilution; ProteinTech Group, Inc.) or a donkey anti-mouse IgG secondary antibody (SA00001-8; 1:5,000 dilution; ProteinTech Group, Inc.). The protein bands were visualized using the Odyssey Western Blot Analysis system (LI-COR Biosciences) and quantified using Image $\mathrm{J}$ version 7.6.5 (National Institutes of Health).

Wound healing and Transwell assays. NCI-H2170 cells were cultured in six-well plates $\left(6 \times 10^{4}\right.$ cells/well) and transiently transfected with the aforementioned specific plasmids. Following $24 \mathrm{~h}$ of transfection $\left(37^{\circ} \mathrm{C}\right)$ the cells were $\sim 100 \%$ confluent, and a linear scratch was created in the cell monolayer using a 200- $\mu$ l pipette tip. The cells were subsequently cultured under standard conditions in serum-free DMEM medium (Procell Life Science and Technology Co., Ltd.) at $37^{\circ} \mathrm{C}$ in the presence of $5 \% \mathrm{CO}_{2}$. Image $\mathrm{J}$ software (version 1.8.0; National Institutes of Health) was used to calculate the wound width. Images of the wound were captured at $24 \mathrm{~h}$ using a light microscope (Nikon Corporation; magnification $\mathrm{x} 100)$. The migration distance was calculated as the width of the scratch at $24 \mathrm{~h}$ minus the width of the scratch at $0 \mathrm{~h}$. The 
A

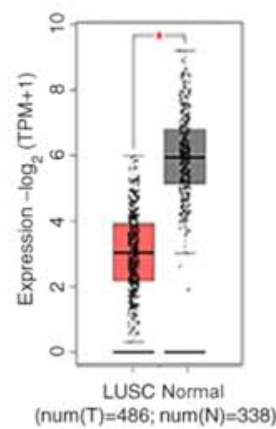

D

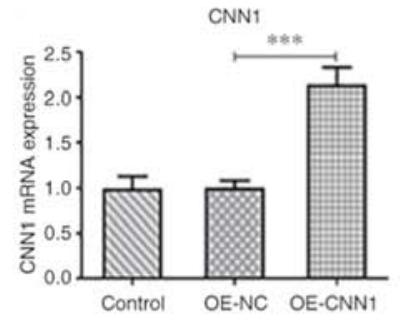

E
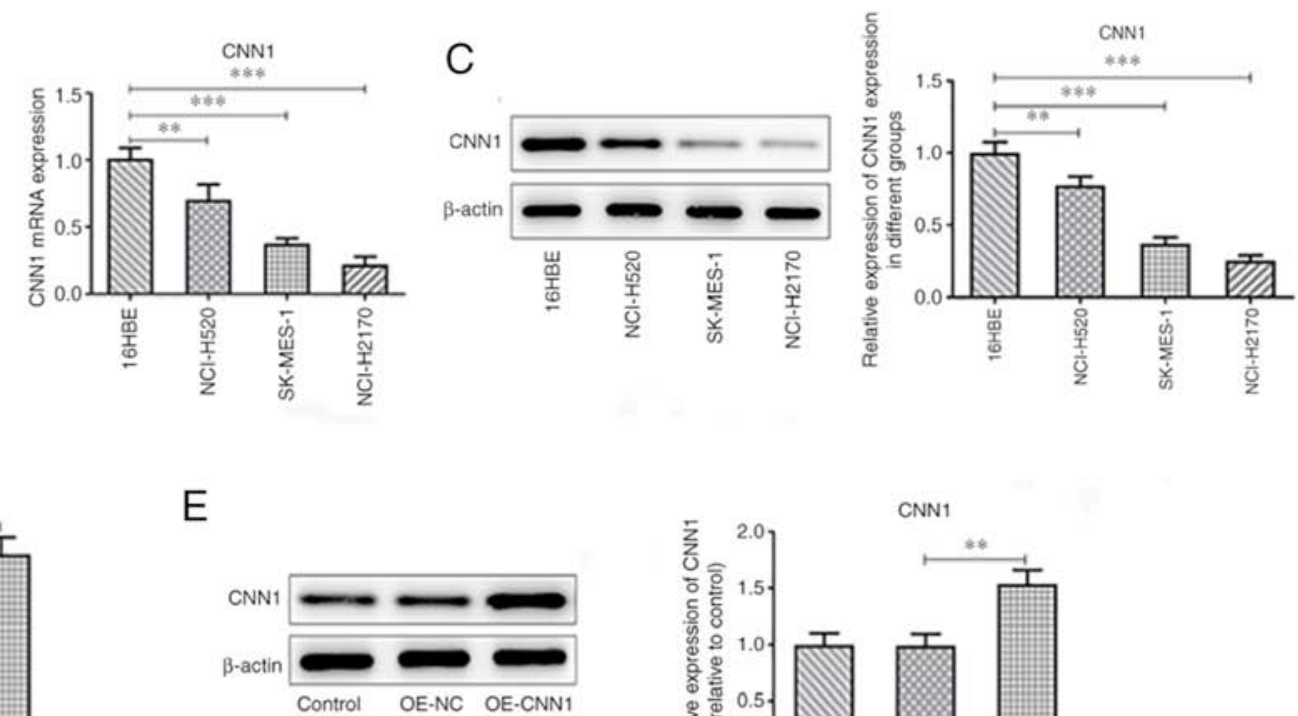

$\mathrm{F}$
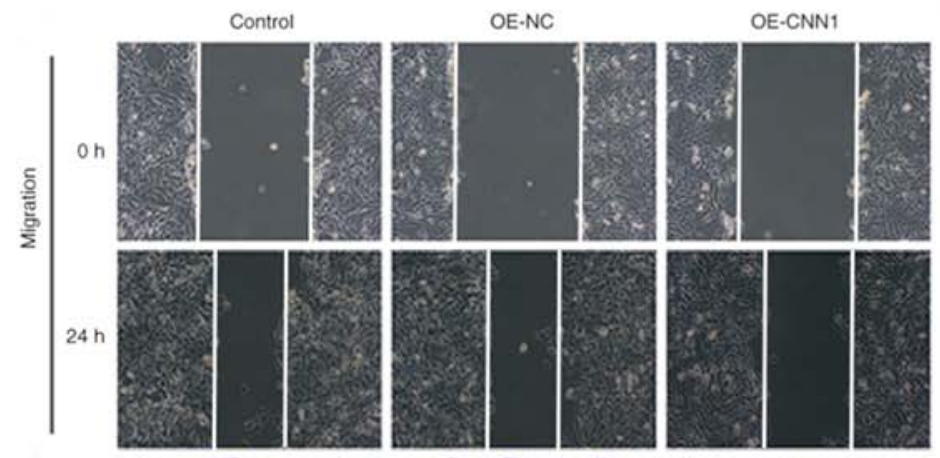

G
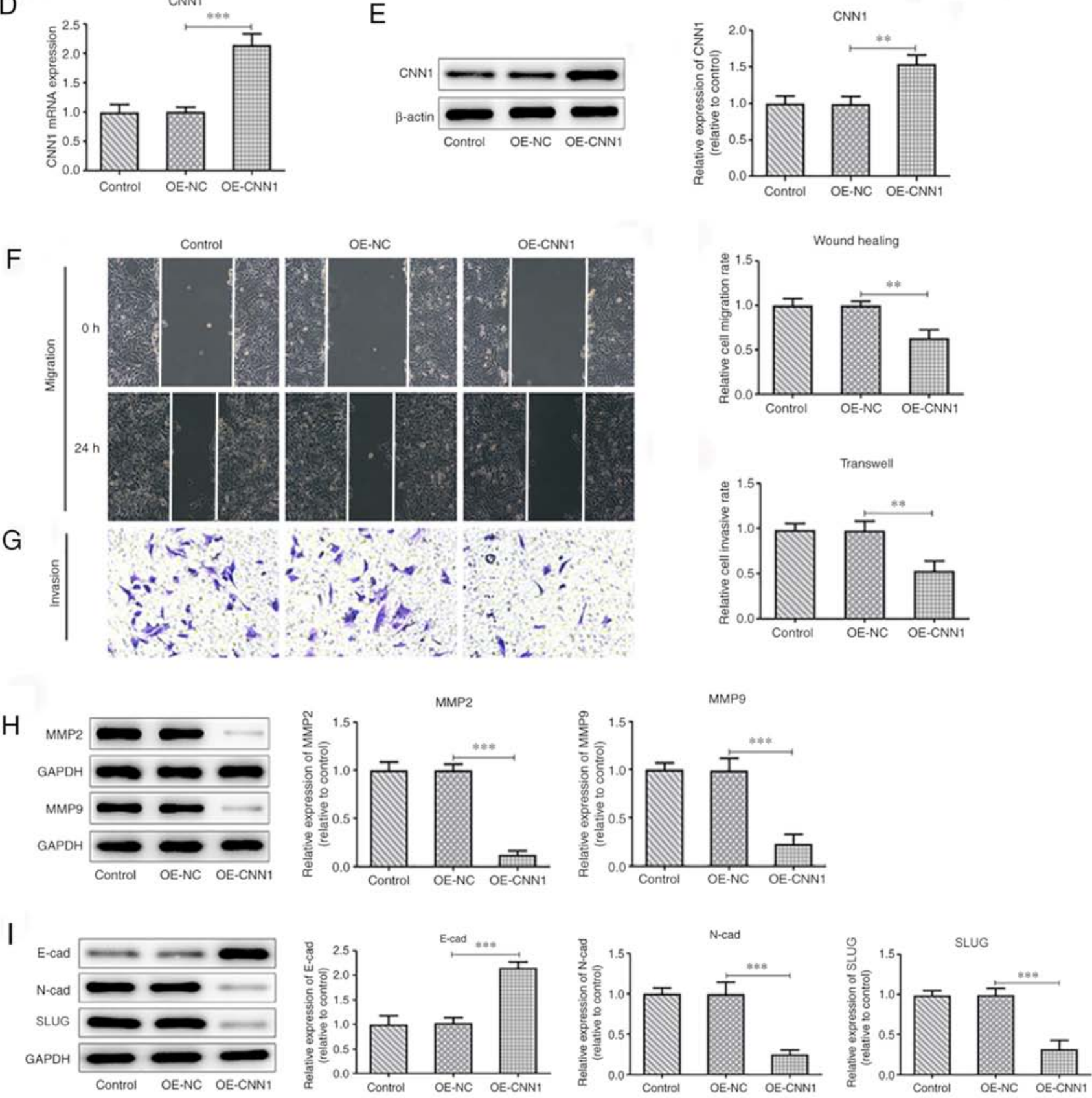

Figure 1. Continued.

relative migration rate was calculated by normalizing to the control group.

The invasive ability of the cells was observed using a Transwell invasion assay. Following $48 \mathrm{~h}$ of treatment, the cells were collected for detection. A total of $200 \mu \mathrm{l}$ cell suspension in serum-free DMEM containing $5 \times 10^{5}$ cells were inoculated in the upper Transwell chamber which was coated with Matrigel at $37^{\circ} \mathrm{C}$ for $30 \mathrm{~min}$ (Corning, Inc.), and cultured at $37^{\circ} \mathrm{C}$ in the 

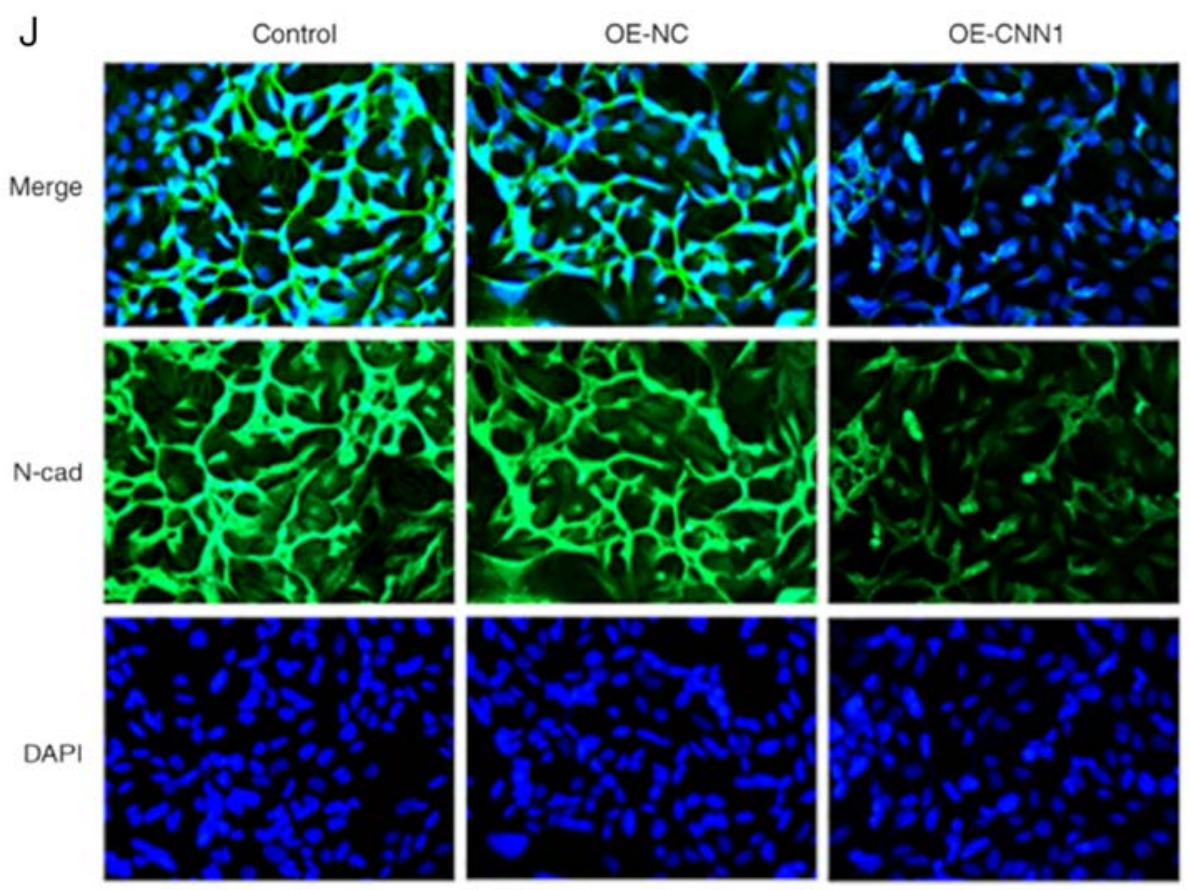

Figure 1. Overexpression of CNN1 inhibits the migration, invasion and EMT of NCL-H2170 cells. (A) GEPIA database predicted the decreased expression of CNN1 in LUSC. (B) CNN1 expression levels in cells were detected using RT-qPCR. (C) CNN1 protein expression levels in the various cell lines were detected using western blotting. (D) Protein expression was quantified using Image J. (E) CNN1 expression levels in NCL-H2170 cells before and after CNN1 overexpression were detected using western blotting and RT-qPCR. (F) Wound healing assays were conducted to evaluate the migratory capacity of NCL-H2170 cells (magnification, x100). (G) Transwell assays were performed to assess the invasive capacity of NCL-H2170 cells (magnification, x100). (H) Western blot analysis was used to detect migration-associated proteins in NCL-H2170 cells. (I) Western blot analysis was used to detect the levels of EMT-associated proteins in NCL-H2170 cells. (J) Immunofluorescence analysis was used to detect the expression levels of N-cad (magnification, x200) in NCL-H2170 cells. ${ }^{*} \mathrm{P}<0.05,{ }^{* *} \mathrm{P}<0.01,{ }^{* * * *} \mathrm{P}<0.001$. All experiments were repeated three times. CNN1, calponin 1; E-cad; E-cadherin; EMT, epithelial-to-mesenchymal transition; LUSC, lung squamous cell carcinoma; N, normal; N-cad, N-cadherin; NC, negative control; OE, overexpression; RT-qPCR, reverse-transcription quantitative PCR; SLUG, zinc finger protein SNAI2; T, tumor; TPM, transcripts per million.

presence of $5 \% \mathrm{CO}_{2}$ for $12 \mathrm{~h}$. DMEM medium containing $10 \%$ FBS was filled into the lower chamber for $24 \mathrm{~h}$. Subsequently, $4 \%$ paraformaldehyde was added for $0.5 \mathrm{~h}$ for fixation at room temperature and the cells were stained with $0.1 \%$ crystal violet for $30 \mathrm{~min}$ at $37^{\circ} \mathrm{C}$. The number of cells passing through the membrane in three random fields was observed under a light microscope with a 100x magnification.

Immunofluorescence. The cells were fixed with $4{ }^{\circ} \mathrm{C} 4 \%$ polyphosphate formaldehyde for $14 \mathrm{~min}$ and permeabilization with 0.1\% Triton X-100 (Beijing Zhongshan Jinqiao Biotechnology Co., Ltd.) for $30 \mathrm{~min}$ at room temperature. This process (permeabilization) was repeated 3 times and the cells were blocked with $10 \%$ goat serum (Beijing Zhongshan Jinqiao Biotechnology Co., Ltd.) for $30 \mathrm{~min}$ at room temperature. Following incubation overnight at $4^{\circ} \mathrm{C}$ with primary antibodies (anti-N-cad; cat. no. ab245117; Abcam; 1:200 dilution), FITC-labeled secondary fluorescent antibodies (goat anti-rabbit IgG; cat. no. ab6717; Abcam; 1:200 dilution) were added to the cells. Following incubation for $0.5 \mathrm{~h}$ at $37^{\circ} \mathrm{C}$, DAPI was added in the dark and the film was sealed and observed using a fluorescence microscope (magnification, x200). Three random fields per sample were analyzed.

Statistical analysis. All experiments were repeated three times. Statistical analysis was performed using GraphPad Prism 5 software (GraphPad Software, Inc.) and all data are presented as mean \pm SEM, unless otherwise specified. Statistical differences between two groups were determined using an unpaired two-tailed Student's t-test, while a one-way ANOVA followed by Tukey's post hoc test were used to analyze data corresponding to more than two groups. $\mathrm{P}<0.05$ was considered to indicate a statistically significant difference.

\section{Results}

Overexpression of CNN1 inhibits cell migration, invasion and EMT. Initially, the GEPIA2 database was used to explore the expression of CNN1 in lung cancer tissues. The results indicated that the expression levels of CNN1 were decreased in LUSC tissues (Fig. 1A). RT-qPCR and western blot analyses were used to detect the expression levels of CNN1 in the following LUSC cell lines: 16HBE, NCI-H520, SK-MES-1 and NCI-H2170 (Fig. 1B-D). The results indicated that the expression levels of CNN1 were the lowest in NCI-H2170 cells. Therefore, these cells were selected for the following experiments. Following successful overexpression of CNN1 (Fig. 1E), cell migratory (Fig. 1F) and invasive (Fig. 1G) rate, protein expression of MMP2 and MMP9 (Fig. 1H) along with N-cad and Slug expression levels (Fig. 1I and J) were decreased, but E-cad was increased, indicating the inhibitory effect of CNN1 overexpression on cell migration, invasion and EMT. 
A

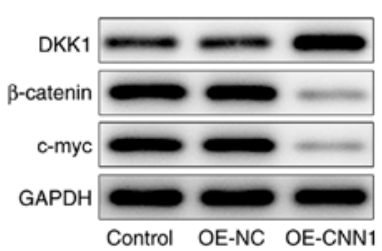

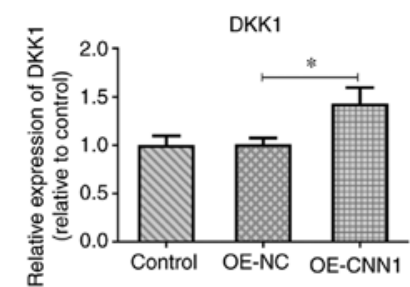
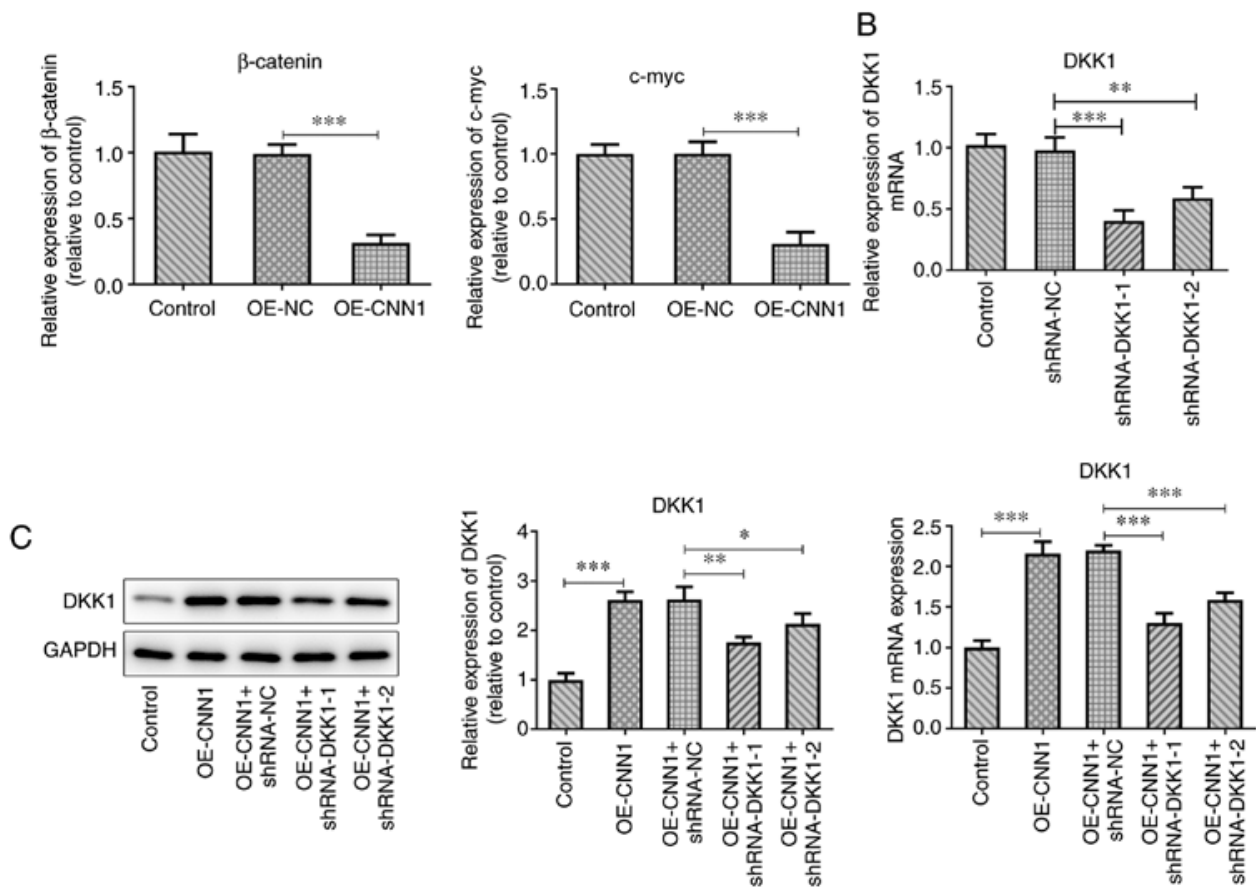

Figure 2. Construction of DKK1 overexpression and DKK1 inference plasmids in NCL-H2170 cells. (A) Expression levels of DKK1, $\beta$-catenin and c-myc protein were detected using western blot analysis. (B) DKK1 knockdown efficiency was verified using RT-qPCR. (C) DKK1 protein and gene expression levels in co-transfected cells were detected using western blot analysis and RT-qPCR. ${ }^{*} \mathrm{P}<0.05,{ }^{* * *} \mathrm{P}<0.01,{ }^{* * * *} \mathrm{P}<0.001$. The experiments were repeated three times. CNN1, calponin 1; DKK1, Dickkopf-1; NC, negative control; OE, overexpression; RT-qPCR, reverse-transcription quantitative PCR; shRNA, short hairpin RNA.

Overexpression of CNN1 inhibits the expression levels of $W n t / \beta$-catenin signaling proteins. The expression levels of DKK1 were upregulated, and those of $\beta$-catenin and c-myc were downregulated, compared with those of the OE-NC group following overexpression of CNN1 (Fig. 2A). An shRNA interference plasmid targeting DKK1 was constructed and the transfection efficacy was confirmed using RT-qPCR (Fig. 2B). The expression levels of DKK1 in shRNA-DKK1 + OE-CNN1 co-transfected cells were detected using RT-qPCR and western blot analyses (Fig. 2C). The mRNA and protein expression levels of CNN1 in the OE-CNN1 + shRNA-DKK1-1 group were lower than those of the OE-CNN1 + shRNA-DKK1-2 group. Therefore, the DKK1-1 group was selected for further experiments.

Interference of DKK1 blocks CNN1 expression and inhibits the migration, invasion and EMT of lung cancer cells. The scratch width, as determined using wound healing assays, was reduced following interference of DKK1 expression compared with that of the plasmid control group (Fig. 3A and C). Corresponding results were observed in the Transwell experiment; interference with DKK1 expression attenuated the ability of increased CNN1 expression levels to inhibit cell invasion (Fig. 3B and C). Following interference with DKK1, the expression levels of MMP2 and MMP9 were increased compared with the OE-CNN1 + shRNA-NC group, as demonstrated using western blot analysis (Fig. 3D). This further suggested that the migratory and invasive ability of the cells in the OE-CNN1 + shRNA-DKK1-1 group was enhanced. The inhibitory effects of CNN1 on the EMT process of lung cancer cells also appeared to be blocked.

The expression levels of $\mathrm{N}$-cad and SLUG along with $\mathrm{N}$-cad fluorescence were increased in the OE-CNN1 + shRNA-DKK1-1 group (Fig. 3E), whereas E-cad expression was decreased (Fig. 3E), when compared with OE-CNN1 + shRNA-NC group.

Interference of TIMP2 inhibits the expression levels of the Wnt/ $\beta$-catenin signaling pathway proteins. GEPIA predicted a positive correlation between CNN1 and TIMP2, with a correlation coefficient of 0.68 (Fig. 4A). It was also predicted that TIMP2 expression was decreased in LUSC tissues (Fig. 4B). The mRNA expression levels of TIMP2 were upregulated following CNN1 overexpression (Fig. 4C). The knockdown efficiency of TIMP2 in cells was detected using RT-qPCR (Fig. 4D and E), and shRNA-TIMP2-1, which was selected for subsequent experiments, exhibited a better knockdown efficiency. CNN1 overexpression increased DKK1 expression level, but reduced $\beta$-catenin and c-myc expression levels (Fig. 4F). Following TIMP2 silencing, the expression levels of 
A
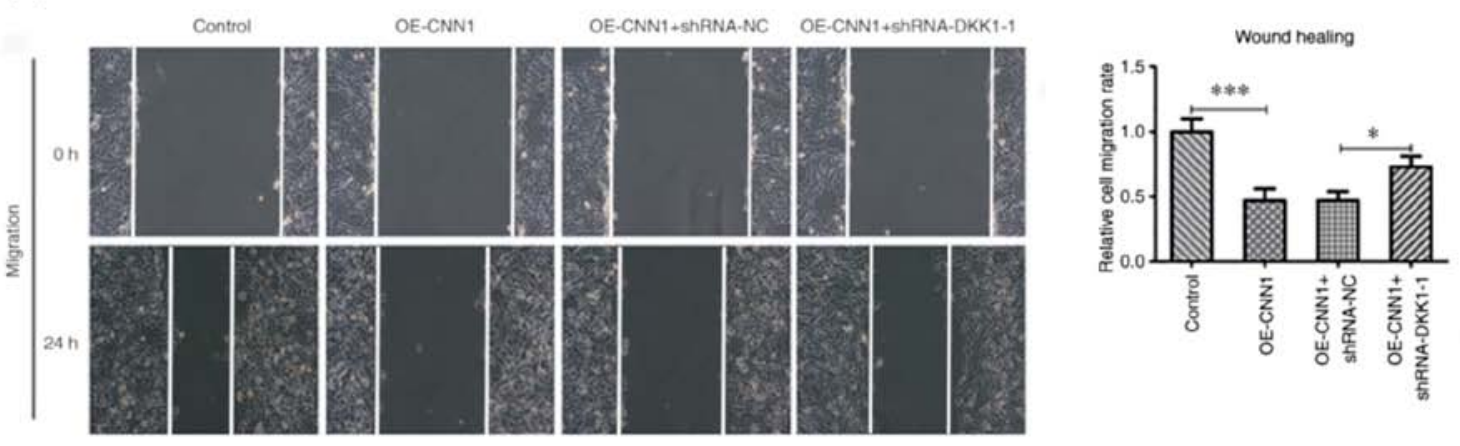

B

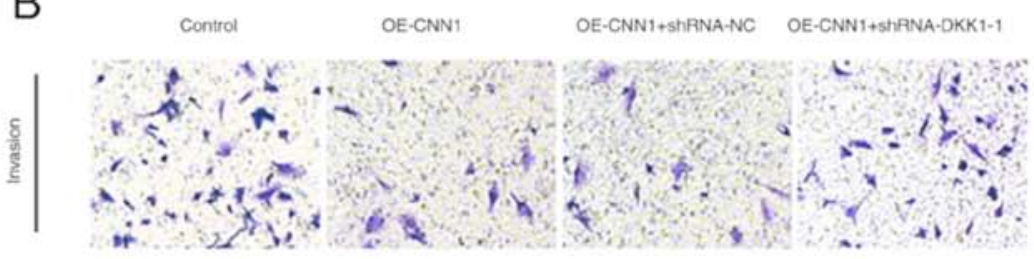

C
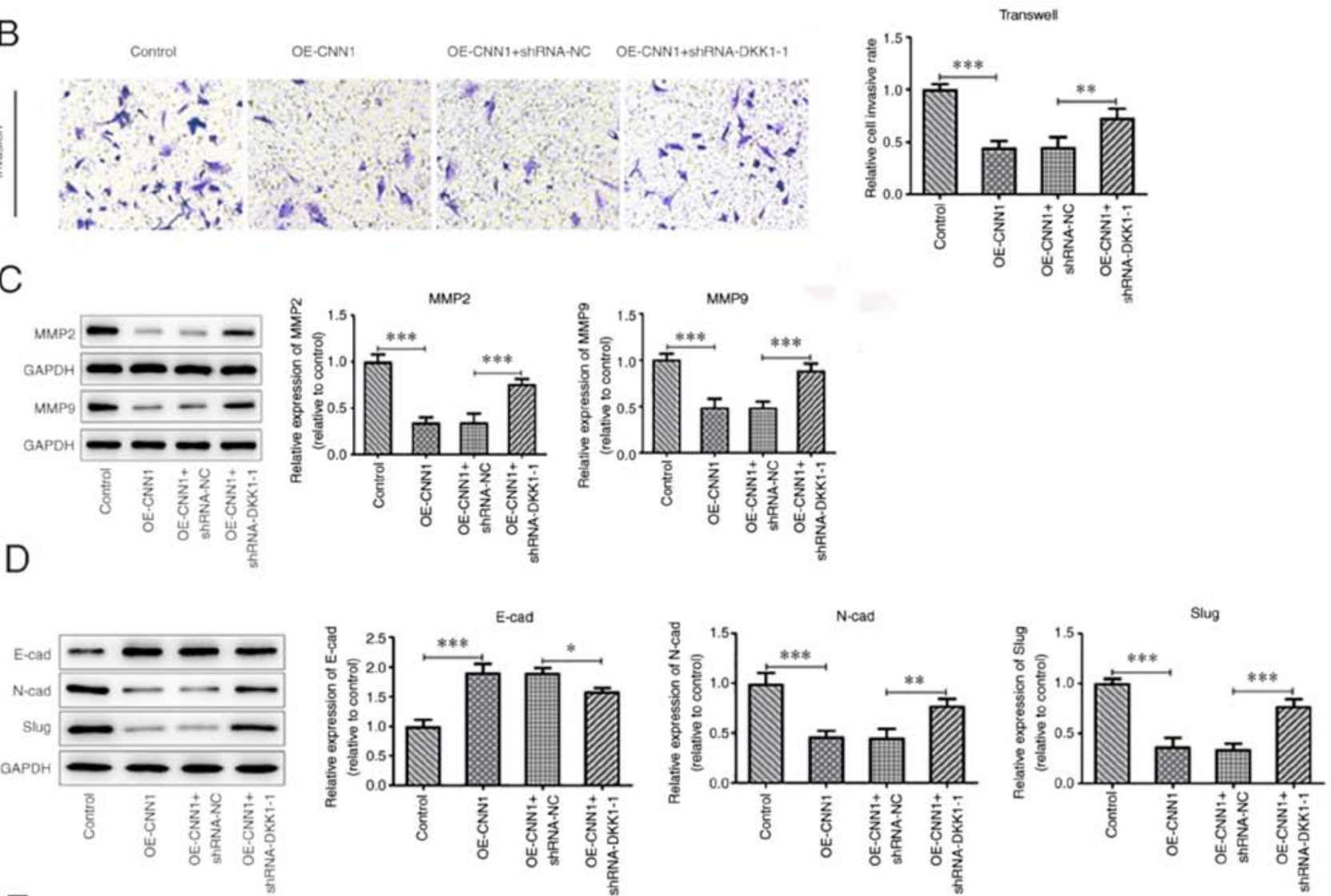

$E$
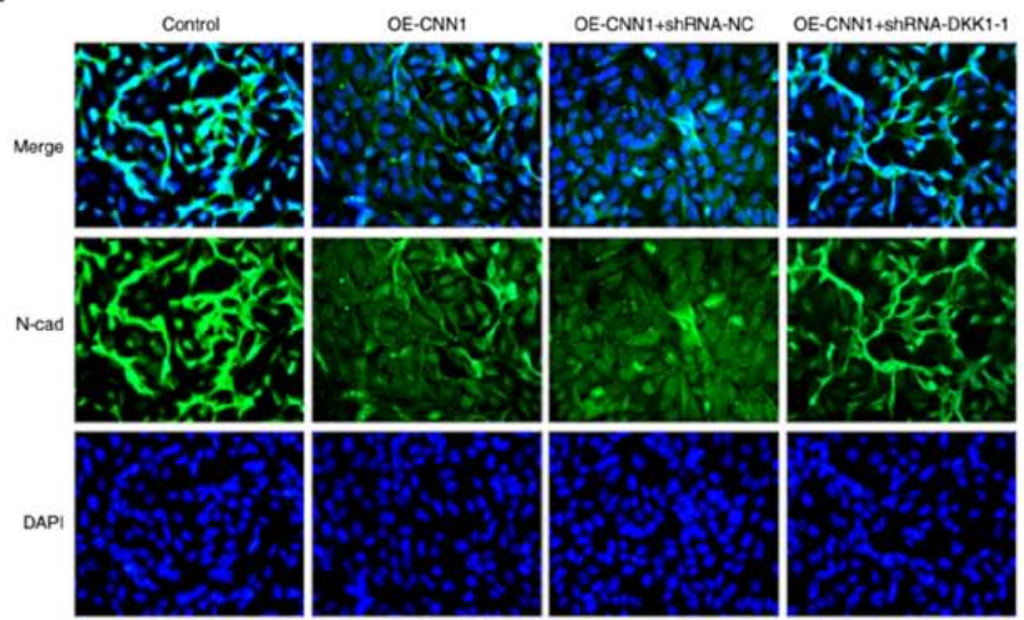

Figure 3. Interference of DKK1 inhibits the migration, invasion and EMT of NCL-H2170 cells. (A) Wound healing assays were conducted to evaluate the migratory capacity of NCL-H2170 cells (magnification, x100). (B) Transwell assays were performed to assess the invasive capacity of NCL-H2170 cells (magnification, $\mathrm{x} 100$ ). (C) MMP2 and MMP9 protein expression levels were detected using western blot analysis. (D) EMT-associated protein expression was detected by western blotting. (E) Immunofluorescence analysis was used to detect the expression levels of $\mathrm{N}$-cad (magnification, $\mathrm{x} 200$ ). ${ }^{*} \mathrm{P}<0.05,{ }^{* *} \mathrm{P}<0.01,{ }^{* * *} \mathrm{P}<0.001$. The experiments were repeated three times. CNN1, calponin 1; DKK1, Dickkopf-1; EMT, epithelial-to-mes-enchymal transition; N-cad, N-cadherin; NC, negative control; OE, overexpression; shRNA, short hairpin RNA; SLUG, zinc finger protein SNAI2. 

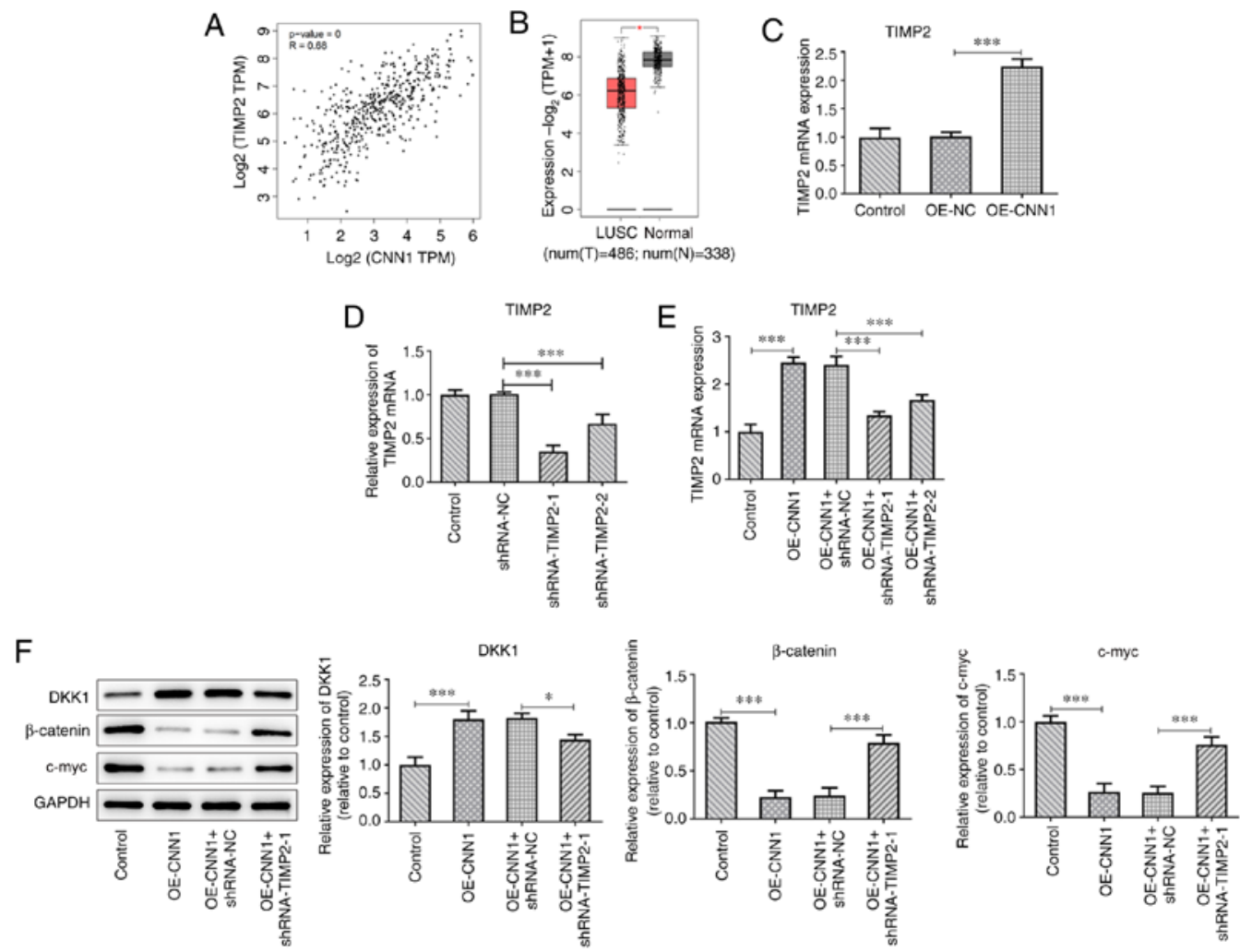

Figure 4. Interference of TIMP2 affects the expression of DKK1-associated proteins in NCL-H2170 cells. (A) GEPIA database prediction of the association between CNN1 and TIMP2. (B) GEPIA database prediction of the expression of TIMP2 in LUSC. (C) TIMP2 expression was detected using RT-qPCR following CNN1 overexpression. (D and E) TIMP2 mRNA expression levels were assessed following transfection with (D) shRNA-TIMP2, as well as (E) OE-CNN1 and shRNA-TIMP2 using RT-qPCR. (F) Protein expression levels of DKK1, $\beta$-catenin and c-myc were detected using western blot analysis. ${ }^{*} \mathrm{P}<0.05$ and ${ }^{* * *} \mathrm{P}<0.001$. The experiments were repeated three times. CNN1, calponin 1; DKK1, Dickkopf-1; LUSC, lung squamous cell carcinoma; $\mathrm{N}$, normal; $\mathrm{NC}$, negative control; OE, overexpression; RT-qPCR, reverse-transcription quantitative PCR; shRNA, short hairpin RNA; T, tumor; TIMP2, tissue inhibitor of metalloproteinases 2; TPM, transcripts per million.

DKK1 were downregulated, and those of $\beta$-catenin and c-myc were upregulated compared with the OE-CNN1 + shRNA-NC group (Fig. 4F).

\section{Discussion}

Lung cancer is a malignant tumor with high morbidity and mortality, which poses a serious threat to human health worldwide (23). NSCLC accounts for $\sim 85 \%$ of all lung cancer cases (24). Significant advances have been made in the treatment of lung cancer (24). Although targeted therapy and immunotherapy have significantly increased the 5-year survival rates, high recurrence rates, metastasis and drug resistance are characteristic of lung cancer (5). Therefore, novel treatments with high efficiency, low cost and low adverse reactions are urgently required.

CNN1 is a known marker of cardiac and smooth muscle cell differentiation. CNN1 is also a member of the cysteine rich 61/connective tissue growth factor/nephroblastoma overexpressed family of growth regulators $(8,25)$. CNN1 is characterized as a pro-angiogenic factor that mediates diverse roles in cell development, proliferation and tumorigenesis (26). However, the association between CNN1 and lung cancer or squamous cell carcinoma development has, to the best of our knowledge, not been previously reported. In the present study, the GEPIA database was used to predict the association between CNN1 and LUSC cells. The expres- sion levels of CNN1 in 16HBE, NCL-H520, SK-MES-1 and NCL-H2170 cells were also detected using western blot analysis. Following overexpression of CNN1, the cell migratory and invasive abilities decreased. Several studies have shown that MMP2 and MMP9 are tumor promoter genes that are highly expressed in a variety of malignant tumor tissue types, such as glioma, lung, liver, colon, pancreatic, breast and cervical cancers $(27,28)$. They promote the formation of new blood vessels in tumors, and accelerate the invasion and metastasis of tumor cells $(27,28)$. In the present study, MMP2 and MMP9 expression levels were downregulated, indicating that overexpression of CNN1 inhibited cell migration. The EMT process involves the transformation of epithelial cells into mesenchymal cells under specific physiological and pathological conditions, resulting in loss of polarity of epithelial cells and intercellular transformation $(29,30)$. The end-result of EMT is cells with mesenchymal cell morphology and properties $(29,30)$. E-cad is a transmembrane glycoprotein widely present in human epithelial cells (31). Its physiological activity is to maintain the adhesion between cells and preserve the integrity of the tissue structure (31). N-cad is a transmembrane protein, which functions to transmit signals. A previous study has shown that the $\mathrm{N}$-cad protein is upregulated in a variety of tumor cell types and that its abnormal expression is associated with the survival, migration, invasion and proliferation of tumor cells (32). The expression levels of 
E-cad and N-cad were downregulated, indicating that overexpression of CNN1 inhibited EMT.

DKK1 is a secretory protein that acts as an inhibitor of the extracellular Wnt signal transduction pathway (19). DKK1 is a member of the DKK protein family and its abnormal regulation is closely associated with the pathogenesis of various types of tumors (19). The Wnt/ $\beta$-catenin pathway is inhibited by degradation of proteasomal $\beta$-catenin, induction of apoptosis and inhibition of cell proliferation (33). Following CNN1 overexpression, DKK1 was upregulated, and $\beta$-catenin and c-myc expression levels were downregulated. Following DKK1 interference, increased cell migratory, invasive and EMT abilities were observed. These results demonstrated that CNN1 could inhibit the invasion, migration and the EMT process of LUSC cells by inactivating the Wnt/ $\beta$-catenin/c-myc signaling pathway. However, the silencing of DKK1 did not block the effects of CNN1 completely, indicating the presence of at least one other pathway that mediates the actions of CNN1 in LUSC, which need to be further explored.

DKK1 is a member of the TIMP family, which is closely associated with lung cancer. TIMP2 can impair the activity of the protease MMP2 to hydrolyze the extracellular matrix, which impedes the process of cell migration and invasion $(19,34)$. The GEPIA database predicted a positive correlation between CNN1 and TIMP2, and TIMP2 expression was found to decrease in LUSC tissues. TIMP2 has been reported to inhibit Wnt signaling to suppress tumor growth and metastasis (18). Therefore, it was speculated that CNN1 exerted its inhibitory effect on the Wnt/ $\beta$-catenin signaling pathway by upregulating TIMP 2 expression. Consistent with this hypothesis, the expression levels of TIMP2 were upregulated following CNN1 overexpression. TIMP2 knockdown blocked the inhibitory effect of CNN1 on the Wnt/ $\beta$-catenin signaling.

In conclusion, $\mathrm{CNN} 1$ regulated the $\mathrm{Wnt} / \beta$-catenin/c-myc signaling pathway by activating TIMP2 to inhibit the invasion, migration and the EMT process in LUSC cells. However, other potential mechanisms need to be uncovered, as well as the usage of other cell lines together with in vivo studies to further clarify the conclusions of the present study.

\section{Acknowledgements}

Not applicable.

\section{Funding}

No funding was received.

\section{Availability of data and materials}

All data generated or analyzed during the present study are included in this published article.

\section{Authors' contributions}

WSL contributed to study conception and design. WSL and XGF contributed to acquisition of data. RML contributed to analysis and interpretation of data. WSL drafted the initial manuscript and revised it critically for important intellectual content. WSL and XGF confirm the authenticity of all the raw data. All authors read and approved the final manuscript.

\section{Ethics approval and consent to participate}

Not applicable.

\section{Patient consent for publication}

Not applicable.

\section{Competing interests}

The authors declare that they have no competing interests.

\section{References}

1. Nasim F, Sabath BF and Eapen GA: Lung Cancer. Med Clin North Am 103: 463-473, 2019.

2. Siegel RL, Miller KD and Jemal A: Cancer statistics, 2016. CA Cancer J Clin 66: 7-30, 2016.

3. Amin MB, Greene FL, Edge SB, Compton CC, Gershenwald JE, Brookland RK, Meyer L, Gress DM, Byrd DR and Winchester DP: The Eighth Edition AJCC Cancer Staging Manual: Continuing to build a bridge from a population-based to a more 'personalized' approach to cancer staging. CA Cancer J Clin 67: 93-99, 2017.

4. Jain D and Roy-Chowdhuri S: Molecular Pathology of Lung Cancer Cytology Specimens: A Concise Review. Arch Pathol Lab Med 142: 1127-1133, 2018.

5. Herbst RS, Morgensztern D and Boshoff C: The biology and management of non-small cell lung cancer. Nature 553: 446-454, 2018.

6. Zhang R, Deng Y, Zhang Y, Zhai GQ, He RQ, Hu XH, Wei DM, Feng ZB and Chen G: Upregulation of HOXA13 as a potential tumorigenesis and progression promoter of LUSC based on qRT-PCR and bioinformatics. Int J Clin Exp Pathol 10: 10650-10665, 2017.

7. Drilon A, Rekhtman N, Ladanyi M and Paik P: Squamous-cell carcinomas of the lung: Emerging biology, controversies, and the promise of targeted therapy. Lancet Oncol 13: e418-e426, 2012.

8. Miano JM, Krahe R, Garcia E, Elliott JM and Olson EN: Expression, genomic structure and high resolution mapping to $19 \mathrm{p} 13.2$ of the human smooth muscle cell calponin gene. Gene 197: 215-224, 1997.

9. Wang X, Wu K, Zhang Z, Lan M, Jin J and Fan D: The effect of calponin and caldesmon in regulation of the gastrointestinal motility during pathophysiological adaptation. Zhonghua Nei Ke Za Zhi 40: 459-462, 2001 (In Chinese).

10. Lv B, Zhao J, Yang F, Huang X, Chen G, Yang K, Liu S, Fan C, $\mathrm{Fu} \mathrm{H}$ and Chen $\mathrm{Z}$ : Phenotypic transition of corpus cavernosum smooth muscle cells subjected to hypoxia. Cell Tissue Res 357: 823-833, 2014.

11. Koganehira Y, Takeoka M, Ehara T, Sasaki K, Murata H, Saida T and Taniguchi S: Reduced expression of actin-binding proteins, $\mathrm{h}$-caldesmon and calponin h1, in the vascular smooth muscle inside melanoma lesions: An adverse prognostic factor for malignant melanoma. Br J Dermatol 148: 971-980, 2003.

12. Sasaki Y, Yamamura H, Kawakami Y, Yamada T, Hiratsuka M, Kameyama M, Ohigashi $\mathrm{H}$, Ishikawa $\mathrm{O}$, Imaoka $\mathrm{S}$, Ishiguro S, et al: Expression of smooth muscle calponin in tumor vessels of human hepatocellular carcinoma and its possible association with prognosis. Cancer 94: 1777-1786, 2002.

13. Yamane T, Asanoma K, Kobayashi H, Liu G, Yagi H, Ohgami T, Ichinoe A, Sonoda K, Wake $\mathrm{N}$ and Kato K: Identification of the critical site of calponin 1 for suppression of ovarian cancer properties. Anticancer Res 35: 5993-5999, 2015.

14. Martín de las Mulas J, Reymundo C, Espinosa de los Monteros A, Millán Y and Ordás J: Calponin expression and myoepithelial cell differentiation in canine, feline and human mammary simple carcinomas. Vet Comp Oncol 2: 24-35, 2004.

15. Clevers $\mathrm{H}$ and Nusse $\mathrm{R}$ : Wnt $/ \beta$-catenin signaling and disease. Cell 149: 1192-1205, 2012.

16. Krishnamurthy N and KurzrockR: Targeting the Wnt/beta-catenin pathway in cancer: Update on effectors and inhibitors. Cancer Treat Rev 62: 50-60, 2018. 
17. Teng $Y$, Wang $X$, Wang $Y$ and Ma D: Wnt/beta-catenin signaling regulates cancer stem cells in lung cancer A549 cells. Biochem Biophys Res Commun 392: 373-379, 2010.

18. Yang S, Liu Y, Li MY, Ng CSH, Yang SL, Wang S, Zou C, Dong Y, Du J, Long X, et al: FOXP3 promotes tumor growth and metastasis by activating Wnt/ $\beta$-catenin signaling pathway and EMT in non-small cell lung cancer. Mol Cancer 16: 124, 2017.

19. Huang Y, Liu L and Liu A: Dickkopf-1: Current knowledge and related diseases. Life Sci 209: 249-254, 2018.

20. Katoh M: Multi-layered prevention and treatment of chronic inflammation, organ fibrosis and cancer associated with canonical WNT/ $\beta$-catenin signaling activation (Review). Int J Mol Med 42: 713-725, 2018.

21. Chanvorachote P, Sriratanasak N and Nonpanya N: C-myc Contributes to Malignancy of Lung Cancer: A potential anticancer drug target. Anticancer Res 40: 609-618, 2020.

22. Livak KJ and Schmittgen TD: Analysis of relative gene expression data using real-time quantitative PCR and the 2(-Delta Delta C(T)) Method. Methods 25: 402-408, 2001.

23. Samarghandian S, Azimi-Nezhad $M$ and Farkhondeh $T$ : Thymoquinone-induced antitumor and apoptosis in human lung adenocarcinoma cells. J Cell Physiol 234: 10421-10431, 2019.

24. Cagle PT, Allen TC and Olsen RJ: Lung cancer biomarkers: Present status and future developments. Arch Pathol Lab Med 137: 1191-1198, 2013.

25. Williams JP, Micoli K and McDonald JM: Calmodulin-an often-ignored signal in osteoclasts. Ann N Y Acad Sci 1192: 358-364, 2010.

26. Menéndez JA, Mehmi I, Griggs DW and Lupu R: The angiogenic factor CYR61 in breast cancer: Molecular pathology and therapeutic perspectives. Endocr Relat Cancer 10: 141-152, 2003.

27. Jackson MT, Moradi B, Smith MM, Jackson CJ and Little CB: Activation of matrix metalloproteinases 2,9 , and 13 by activated protein $\mathrm{C}$ in human osteoarthritic cartilage chondrocytes. Arthritis Rheumatol 66: 1525-1536, 2014.
28. Milner JM, Rowan AD, Cawston TE and Young DA: Metalloproteinase and inhibitor expression profiling of resorbing cartilage reveals pro-collagenase activation as a critical step for collagenolysis. Arthritis Res Ther 8: R142, 2006.

29. Lee HM, Hwang KA and Choi KC: Diverse pathways of epithelial mesenchymal transition related with cancer progression and metastasis and potential effects of endocrine disrupting chemicals on epithelial mesenchymal transition process. Mol Cell Endocrinol 457: 103-113, 2017.

30. Hao Y, Baker D and Ten Dijke P: TGF- $\beta$-mediated epithelial-mesenchymal transition and cancer metastasis. Int J Mol Sci 20: 2767, 2019.

31. Luo T, Yan A, Liu L, Jiang H, Feng C, Liu G, Liu F, Tang D and Zhou T: In vitro study of joint intervention of E-cad and Bmi-1 mediated by transcription activator-like effector nuclease in nasopharyngeal carcinoma. Zhong Nan Da Xue Xue Bao Yi Xue Ban 43: 229-239, 2018 (In Chinese).

32. Liu Y, Yang F, Liang S, Liu Q, Fu S, Wang Z, Yang C and Lin J: N-Cadherin Upregulation Promotes the Neurogenic Differentiation of Menstrual Blood-Derived Endometrial Stem Cells. Stem Cells Int 2018: 3250379, 2018.

33. Igbinigie E, Guo F, Jiang SW, Kelley C and Li J: Dkk1 involvement and its potential as a biomarker in pancreatic ductal adenocarcinoma. Clin Chim Acta 488: 226-234, 2019.

34. Jaschke N, Hofbauer LC, Göbel A and Rachner TD: Evolving functions of Dickkopf-1 in cancer and immunity. Cancer Lett 482: 1-7, 2020.

\footnotetext{
This work is licensed under a Creative Commons Attribution-NonCommercial-NoDerivatives 4.0 International (CC BY-NC-ND 4.0) License.
} 